\title{
Estratégias de Auto-Regulação em Pré-Adolescentes e Adolescentes: Versão Portuguesa do TESQ-E
}

\author{
Self-Regulatory Strategies in Pre-Adolescents and Adolescents: \\ Portuguese Version of TESQ-E
}

\author{
Tânia Gaspar ${ }^{*}$, a Gina Quinas Tomé ${ }^{b}$, Celeste Simões ${ }^{c} \&$ Margarida Gaspar de Matos $^{c}$ \\ ${ }^{a}$ Universidade Lusiada de Lisboa, Lisboa, Portugal, \\ ${ }^{b}$ Faculdade de Ciências Médicas, Universidade Nova de Lisboa, Lisboa, Portugal \\ \& ${ }^{c}$ Universidade de Lisboa, Lisboa, Portugal
}

\begin{abstract}
Resumo
A auto-regulação tem um papel fundamental no comportamento alimentar em crianças e adolescentes face às "tentações" do ambiente. O Tempest Self-Regulation Questionnaire for Eating (TESQ-E; www. tempestproject.eu) é um instrumento de medida da auto-regulação transcultural que foi construído, traduzido e adaptado em nove países europeus, no âmbito do Projeto TEMPEST. Este artigo tem como objetivo o estudo da validação da versão Portuguesa do instrumento. Participaram da amostra 1200 pré-adolescentes e adolescentes Portugueses, com média de idades de 12,5 anos $(D P=1,61)$, entre os nove e os 17 anos de idade, $48,3 \%$ rapazes, de diferentes níveis de escolaridade $5^{\circ}$ ano $(16,8 \%), 6^{\circ}$ ano $(19,8 \%), 7^{\circ}$ ano $(30,8 \%), 8^{\circ}$ ano $(17,8 \%)$ e $9^{\circ}$ ano $(14,9 \%)$. A versão portuguesa do instrumento demonstrou boas propriedades métricas, e confirmou a estrutura factorial da versão original: três dimensões, que incluem seis estratégias de auto-regulação, duas em cada dimensão. A dimensão mais popular foi a Manutenção de objectivos de alimentação saudável, e a menos popular, Mudança do sentido atribuído às tentações. Conclui-se que o TESQ-E é um instrumento confiável para estimar as estratégias de auto-regulação em crianças e adolescentes. Orientações psicométricas são apresentadas para a população portuguesa entre os nove e os 17 anos.

Palavras-chave: Auto-regulação, adolescentes, comportamento alimentar, obesidade, promoção da saúde.

Abstract

Self-regulation plays a key role in the eating behavior of children and adolescents, while facing the environment "temptations". The Tempest Self-Regulation Questionnaire (TESQ-E; www.tempestproject.eu) is an instrument for measuring cross-cultural self-regulation. It has been constructed, adapted and translated into nine European Countries within the TEMPEST project. This article aims to study the validation of the Portuguese version of the instrument. The sample includes 1200 Portuguese pre-adolescents and adolescents, of average age 12.5 years $(S D=1.61)$ between nine and 17 years of age, $48.3 \%$ boys, from different educational grades: $5^{\text {th }}$ grade $(16.8 \%), 6^{\text {th }}$ grade $(19.8 \%), 7^{\text {th }}$ grade $(30.8 \%), 8^{\text {th }}$ grade $(17.8 \%)$ and $9^{\text {th }}$ grade $(14.9 \%)$. The Portuguese version of the instrument demonstrated good psychometric properties and kept the factor structure of the original version: three dimensions, which include six strategies for self -regulation, two in each dimension. The most popular dimension was Maintaining healthy eating objectives, and the less popular Change the meaning of temptation. The study concluded that the TESQ-E is a reliable instrument to estimate the self-regulation strategies in children and adolescents. Psychometric guidelines are presented to the Portuguese population aged nine to 17 years old.

Keywords: Self-regulation, adolescents, eating behavior, obesity, health promotion.
\end{abstract}

As diversas definições do constructo auto-regulação incluem duas propriedades básicas da auto-regulação (Cameron \& Leventhal, 2003). A primeira diz respeito ao facto da auto-regulação ser um sistema motivacional e di- nâmico de estabelecimento de objectivos, desenvolvimento e implementação dos mesmos, avaliação dos progressos e revisão dos objectivos e estratégias. A segunda men-
*Endereço para correspondência: Universidade de Lisboa, Faculdade de Motricidade Humana, Estrada da Costa, Lisboa, Portugal 1349-008. E-mail: tania.gaspar.barra@ gmail.com, ginatome@sapo.pt, csimoes@fmh.ulisboa.pt e mmatos@fmh.ulisboa.pt
Agradecimentos: Este estudo integrado no consórcio TEMPEST foi financiado pelo $7^{\circ}$ Programa Quadro/ Comunidade Europeia (Health-F2-2008-223488). Os autores agradecem o apoio de todos os membros do Consórcio TEMPEST.

Cópia gratuita do instrumento no site do Projecto TEMPEST: www.tempestproject.eu 
ciona que auto-regulação é também um manuseamento de respostas emocionais, que são vistas como elementos cruciais do sistema motivacional e estão intrinsecamente relacionadas com o processo cognitivo (De Ridder \& de Wit, 2006).

Segundo Baumeister (1999) o ser humano tem capacidade para resistir aos seus impulsos, adaptar os seus comportamentos e mudá-los para atingir os seus objectivos. A auto-regulação refere-se aos vários processos através dos quais a mente humana exerce controlo sobre as suas funções, os seus estados e os seus processos internos (Vohs \& Baumeister, 2004), ou seja, aos esforços que o ser humano faz para alterar os seus pensamentos, sentimentos, desejos/ vontades e acções em função dos muitos objectivos que pretende alcançar (Vohs \& Baumeister, 2004). Por isso, auto-regulação é um aspecto vital de adaptação humana, em que o indivíduo, para não se tornar um mero espectador dos acontecimentos de vida, tem de ser um agente activo de tomadas de decisão (Baumeister, 2005).

Vários estudos mostram que a capacidade para regular as acções se desenvolve gradualmente durante a infância e a adolescência (Sokol, Grouzet, \& Muller, 2013). Estes períodos requerem adaptações ao contexto e vice-versa, sendo este um processo bi-direccional de regulação desenvolvimental (Gestsdottir \& Lerner, 2008). Quando aplicado a crianças mais novas o termo auto-regulação é utilizado para referir várias capacidades, como por exemplo, alternar rapidamente entre tarefas diferentes, focar a atenção ou controlar as emoções. Por outro lado, a auto-regulação mede a capacidade dos adolescentes para monitorizar as suas actividades, avaliar os seus desempenhos, motivarem-se e manter a resiliência enquanto vivenciam experiências de desilusão escolares e sociais (Zimmerman, 2002).

A auto-regulação geralmente é construída como um processo dinâmico que envolve esforços conscientes que influenciam pensamentos, sentimentos e comportamentos para atingir um objectivo num contexto de mudança ambiental (Zeidner, Booekaerts, \& Pintrinch, 2000). A auto-regulação transmite envolvimentos individuais de manuseamento dos seus próprios processos de mudança (Abraham, Norman, \& Conner, 2000), incluindo a consideração consciente da importância da competição entre objectivos e a priorização de objectivos em particular (Abraham \& Sheeran, 2000).

Segundo Duckworth e Seligman (2006) a auto-regulação e o auto-controlo são cruciais para o sucesso do indivíduo em várias áreas. Estes autores referiram que estudantes com maior auto-controlo têm um melhor ajustamento psicológico, melhores relações interpessoais e um melhor desempenho em tarefas, da mesma forma que $o$ auto-controlo parece ser o melhor preditor para 0 desempenho académico. No mesmo sentido, Baumeister e Vohs (2004) verificaram que pessoas com elevado auto-controlo apresentam um menor uso de substâncias e de álcool, menores índices de crime e delinquência, melhor avaliação da saúde e melhores comportamentos de saúde.
A maioria dos adolescentes não tem os seus objectivos relativos à manutenção da saúde definidos claramente da mesma forma que os adultos, isto porque a doença é relativamente rara em jovens. No entanto, isto não significa que os jovens não tenham objectivos de saúde, apenas não são tão relevantes. Estes objectivos podem existir num sentido abstracto, mas têm pouca probabilidade de influenciar as acções diárias. As respostas de muitos adolescentes que têm comportamentos de risco sugerem, de forma directa ou indirecta, de que não planearam o comportamento antes deste acontecer (Gebhardt, 2006).

Algumas situações de obesidade e outras perturbações do comportamento alimentar refletem uma incapacidade de parar de comer, especialmente alimentos ricos em gorduras, tal como a incapacidade de resistir a tentações (De Vet et al., 2013). As influências sociais no comportamento alimentar são extremamente poderosas, ultrapassando muitas vezes outras influências, incluindo as principais intenções do indivíduo ou os seus objectivos. Muitas vezes os indivíduos parecem utilizar as companhias de refeição como modelos para a quantidade e qualidade de comida que ingerem (Luszcznska et al., 2013). A auto-regulação nas crianças e adolescentes tem um importante papel no comportamento alimentar e nas estratégias de lidar com as tentações sociais e do ambiente (Gaspar, Matos, Luszczynska, Baban, \& de Wit, 2014).

O objetivo do presente estudo é a validação da versão em português do questionário Tempest Self-Regulation Questionnaire for Eating (TESQ-E; www.tempestproject. eu), suas propriedades psicométricas e sua capacidade de identificar diferenças de género e desenvolvimentais, numa população juvenil portuguesa.

\section{Metodologia}

\section{Instrumento}

O TESQ-E é um instrumento transcultural que mede a auto-regulação no âmbito do comportamento alimentar em crianças e adolescentes. A versão original foi criada em inglês no âmbito do consorcio TEMPEST (www. tempestproject.eu) e cada país traduziu e retro-traduziu para língua nativa do país. A versão final tem 24 itens, que devem ser respondidos através de uma escala de Likert de cinco pontos, variando de 1 (nunca) a 5 (sempre). O Instrumento está organizado em três dimensões (Estratégias que atuam face às tentações diretamente; Estratégias que atuam face ao significado da tentação e Estratégias que atuam diretamente face aos objetivos). Cada dimensão inclui duas estratégias que são avaliadas com quatro itens cada, e uma pontuação média foi calculada para cada dimensão (Stok, De Ridder, de Vet, de Wit, \& the Tempest Consortium, 2013).

A primeira dimensão Redução das tentações, reflete estratégias para lidar com o ambiente de alimentos diretamente e inclui itens que descrevem o controlo da tentação (por exemplo: Garantir que as batatas fritas 
Gaspar, T., Tomé, G. Q., Simões, C. \& Matos, M. G. (2015). Estratégias de Auto-Regulação em Pré-Adolescentes e Adolescentes: Versão Portuguesa do TESQ-E.

estão arrumadas na dispensa, longe da vista, enquanto se vê televisão) e o evitamento da tentação (exemplo: Evitar o corredor dos doces no supermercado; alfa de Cronbach $=0,83)$.

A segunda dimensão, Mudança do sentido atribuído às tentações, reflete estratégias para mudar o significado do ambiente alimentar, e inclui itens que descrevem distração (por exemplo: Quando tiver vontade de comer doces, tentar encontrar outra coisa para fazer) e a supressão (exemplo: Se quiser comer coisas não saudáveis, dizer "não" a si mesmo; alfa de Cronbach $=0,86$ ).

A terceira dimensão Manutenção de objetivos de alimentação saudável, reflete estratégias para lidar com o objetivo de comer de forma saudável e inclui itens que descrevem o Definição de objetivos e regras (por exemplo: Ter um acordo consigo mesmo sobre quantos doces pode ter por dia) e Reflexão sobre os objetivos (exemplo: "Se eu estiver a comer demasiado, penso na forma como esse comportamento pode prejudicar a prática de exercício físico"; alfa de Cronbach =0,86). Os itens das 3 dimensões apresentam na versão original, um correlação elevada e estatisticamente significativa (De Vet et al., 2014; Stok et al., 2013).

\section{Procedimento}

Tradução. A tradução do instrumento do Inglês para Português foi feito por meio de um procedimento de back-translation: Um investigador traduziu os itens para a língua nacional. Outro investigador traduziu os itens de volta da língua nacional para o inglês. Um terceiro investigador comparou as duas versões, e as decisões finais sobre as formulações de linguagem, neste procedimento, são feitas por todos, resultando numa última versão em língua Portuguesa.

Recolha de Dados. O protocolo de recolha de dados cumpriu as diretrizes éticas e foi obtido o consentimento dos participantes e dos seus pais. O estudo foi submetido à Comissão de Ética do Hospital São João e seguiu todas as diretrizes e normas éticas da investigação com humanos. O estudo preserva o anonimato pelo que em Portugal não necessita da aprovação da comissão de protecção de dados. A amostra foi de conveniência e a distribuição das escolas foi realizada tendo em consideração escolas com estatuto socio-económico (ESE) alto e ESE baixo seguiram a instrução do estudo internacional para todos os países (De Vet et al., 2014).

A turma foi a unidade de análise, foi usada uma amostra de conveniência incluindo escolas de zona rural e urbana e escolas em zonas prioritariamente de ESE médio /alto e escolas em zonas prioritariamente de ESE baixo.

Os participantes, com idades entre 10 e 17 anos foram convidados a preencher o questionário numa sessão na escola, em contexto de sala de aula. $\mathrm{O}$ preenchimento do questionário levou cerca de 30 minutos. $\mathrm{O}$ instrumento incluiu variáveis sócio-demográficas, e estratégias de auto-regulação e ainda outras questões relacionadas com o comportamento alimentar, fora do âmbito desta validação. O processo de recolha de dados levou cerca de 45 minutos em cada uma das turmas.

\section{Participantes}

O estudo envolveu 1200 crianças e adolescentes, com idade média de 12,5 anos $(D P=1,61)$, variando de 9 a 17 anos, foram estabelecidos dois grupos etários: pré-adolescentes- 9 - 12 anos (50,7\%) e adolescentes- 13 - 17 anos de idade $(49,3 \%)$, com média de índice de massa corporal (IMC) $19,98(D P=3,67)$, variando de 11,87 a $56,57,48,3 \%$ rapazes, de diferentes níveis de ensino: $5^{\circ}$ ano $(16,8 \%)$, $6^{\circ}$ ano $(19,8 \%), 7^{\circ}$ ano $(30,8 \%), 8^{\circ}$ ano $(17,8 \%)$ e $9^{\circ}$ ano $(14,9 \%), 23 \%$ apresentam um ESE baixo e $77 \%$ apresentam um ESE médio/alto. Cerca de $70 \%$ reside numa zona urbana e $30 \%$ numa zona rural. A maioria das crianças e adolescentes falam português com os pais $(92,6 \%)$ e com os amigos (98\%).

\section{Análise de Dados}

Os dados foram analisados usando SPSS versão 20 . Para análise dos dados foram realizados análise da consistência interna através do teste de Alfa de Cronbach, Análise das correlações, comparação das médias entre género e idade, através do teste ANOVA. Para a realização da análise fatorial confirmatória proposta, utilizou-se o modelo de equações estruturais (Structural Equations Modeling [SEM]), através do programa estatístico EQS, (Structural Equation Modeling Software), versão 6.1.

\section{Resultados}

\section{Análise Consistência Interna}

Mantendo-se a estrutura fatorial inicial proposta pelos autores (De Vet et al., 2014; Stok et al., 2013): 3 dimensões com 2 tipos de estratégias para cada dimensão, a escala TESC-E mostrou uma boa consistência interna para todos os três fatores de ordem superior (Dimensões): $\alpha=$ 0,81 na Redução de tentações $\alpha=0,85$ na Mudança do sentido atribuído a tentações e $\alpha=0,86$ na Manutenção de objetivos de alimentação saudável, sendo a dimensão mais frequente a Manutenção de objectivos de alimentação saudável. E a menos frequente a Mudança do sentido atribuido às tentações.

Verificou-se também uma boa consistência interna para todos os 6 fatores / estratégias, com valores entre $\alpha=0,69$ em Evitamento e $\alpha=0,79$ em Definição de objetivos e regras, sendo a estratégia mais frequente a Reflexão sobre os objetivos e a menos popular a Supressão.

\section{Análise Factorial Confirmatória}

Foram testados dois modelos diferentes, analisando separadamente as subescalas que constituem o questionário TESQ-E (ver Figura 1 e Figura 2).

$\mathrm{Na}$ realização do modelo de análise fatorial I, os resultados obtidos relativamente à adequação do modelo 
explicativo proposto mostraram que este apresentou bons níveis de adequação (ver Tabela 2). Os resultados obtidos no Wald test, não revelaram a existência de ligações não significativas (Bentler, 1995). Assim, optou-se por não se fazer alterações ao modelo inicial, que revelou bons índices de ajustamento, apresentando o modelo boa adequação (ver Tabela 2).

A solução estandardizada obtida no modelo de análise fatorial confirmatória I (Figura 1) permite verificar que no geral o fator independente tem uma boa saturação nos factores dependentes que variam entre $\beta=0,81 \mathrm{e}$ $\beta=0,93$.
A variância explicada dos factores, bem como os residuais, relativamente ao modelo de análise fatorial confirmatório I, são apresentados na Tabela 3. Pode observar-se que esses valores são igualmente adequados e variam entre $R^{2}=0,65$ e $R^{2}=0,86$.

Relativamente ao modelo de análise fatorial II. Também neste modelo, os resultados obtidos para adequação do modelo explicativo proposto mostraram que este apresentou bons níveis de adequação (ver Tabela 2). Os resultados obtidos no Wald test, não revelaram a existência de ligações não significativas.

Tabela 1

Dados Descritivos, Consistência Interna das Três Dimensões do Questionário

\begin{tabular}{ccccccc}
\hline Dimensões & Estratégias & $\begin{array}{c}\text { No. } \\
\text { Itens }\end{array}$ & $N$ & $M$ & $D P$ & Cronbach \\
\hline Redução das tentações & $\begin{array}{c}\text { Evitamento } \\
\text { Controlo }\end{array}$ & 8 & 1089 & 18,81 & 6,96 & 0,806 \\
$\begin{array}{c}\text { Mudança do sentido } \\
\text { atribuido às tentações }\end{array}$ & $\begin{array}{c}\text { Distração } \\
\text { Supressão }\end{array}$ & 8 & 1098 & 18,10 & 6,96 & 0,846 \\
$\begin{array}{c}\text { Manutenção de objetivos } \\
\text { de alimentação saudável }\end{array}$ & $\begin{array}{c}\text { Definição de objetivos e regras } \\
\text { Reflexão sobre os objetivos }\end{array}$ & 8 & 1092 & 19,87 & 7,76 & 0,861 \\
Estratégias & Itens & No. & Itens & & & \\
Evitamento & A17, A18, A19, A20 & 4 & 1131 & 9,00 & 3,82 & 0,693 \\
Controlo & B21, B22, B2, B24 & 4 & 1125 & 9,88 & 3,98 & 0,701 \\
Distração & E52, E53, E54, E55 & 4 & 1117 & 9,18 & 3,84 & 0,733 \\
Supressão & F56, F57, F58, F60 & 4 & 1132 & 8,93 & 3,76 & 0,774 \\
Definição de objetivos e regras & D46, D47, D48, D50 & 4 & 1119 & 9,22 & 4,11 & 0,793 \\
Reflexão sobre os objetivos & C31, C33, C35, C38 & 4 & 1138 & 10,64 & 4,36 & 0,779 \\
\hline
\end{tabular}

Tabela 2

Indices de Ajustamento

\begin{tabular}{lccccc}
\hline & $\chi 2(g l)^{1}$ & $\mathrm{CFI}^{2}$ & $\mathrm{NNFI}^{3}$ & RMSEA $^{4}(90 \%$ IC) & SRMR $^{5}$ \\
\hline Análise Fatorial Confirmatória I & $598,1594(246)$ & 0,95 & 0,95 & $0,038(0,034-0,042)$ & 0,038 \\
Análise Fatorial Confirmatória II & $545,8723(244)$ & 0,96 & 0,95 & $0,036(0,032-0,040)$ & 0,036 \\
\hline
\end{tabular}

Notas. ${ }^{1}$ Chi-Square $;{ }^{2}$ Comparative Fit Index $;{ }^{3}$ Non Normed Fit Index; ${ }^{4}$ Root Mean Square Error Aproximation; ${ }^{5}$ Standardized Root Mean Residual. 
Gaspar, T., Tomé, G. Q., Simões, C. \& Matos, M. G. (2015). Estratégias de Auto-Regulação em Pré-Adolescentes e Adolescentes: Versão Portuguesa do TESQ-E.

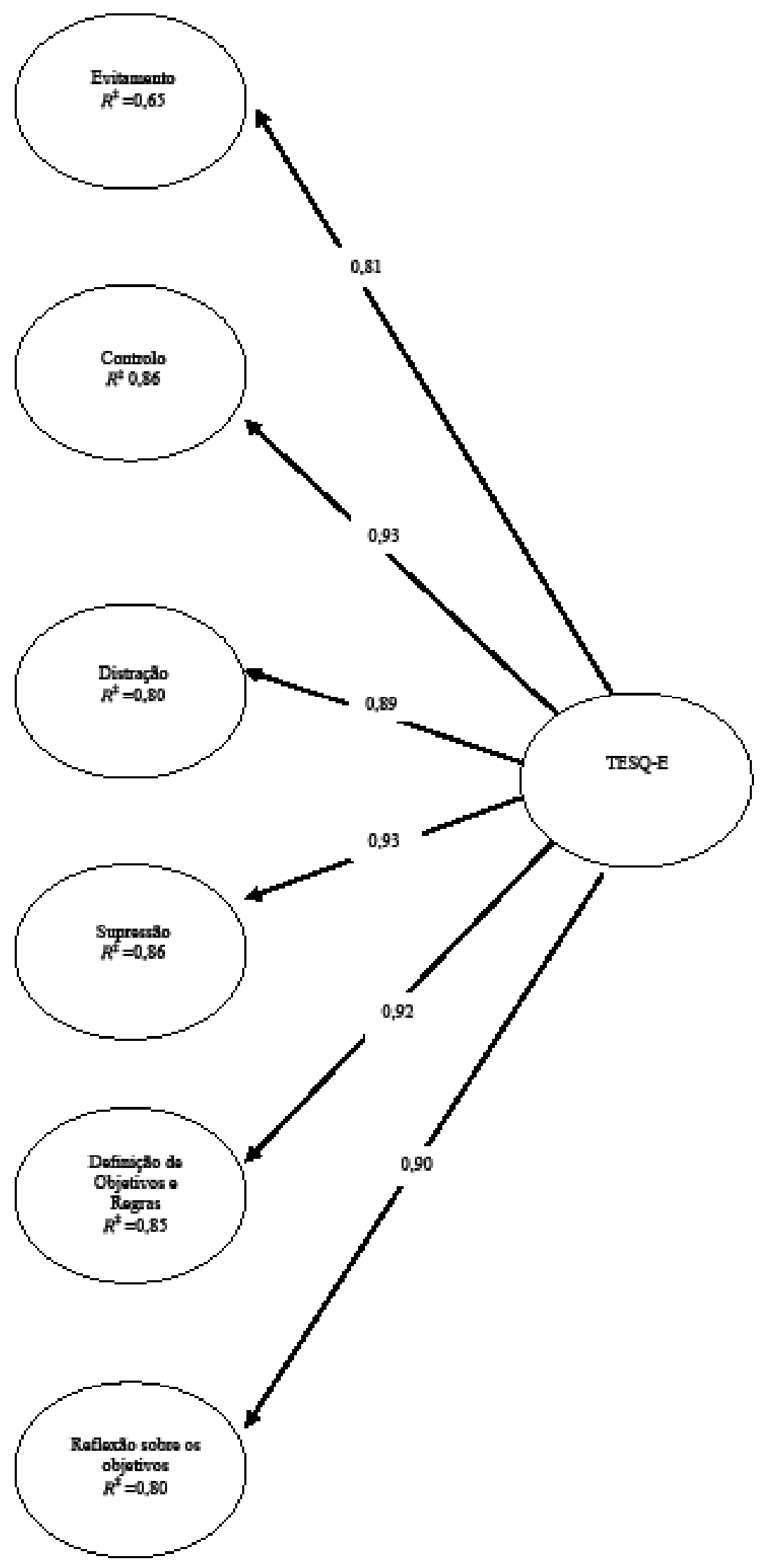

Figura 1. Análise fatorial confirmatória I. 
A solução estandardizada obtida no modelo de análise fatorial confirmatória II (Figura 2) manteve valores semelhantes ao modelo I, revelando que os fatores independentes têm grande saturação nos factores dependentes (entre $\beta=0,87$ e $\beta=1$ ).

As correlações entre os factores independentes variam entre 0,88 e 0,97 (Tabela 4).
A variância explicada dos factores, bem como os residuais, relativamente ao modelo de análise fatorial confirmatório II, são apresentados na tabela.

Pode-se observar que esses valores são igualmente adequados e variam entre $R^{2}=0,76$ e $R^{2}=1$.

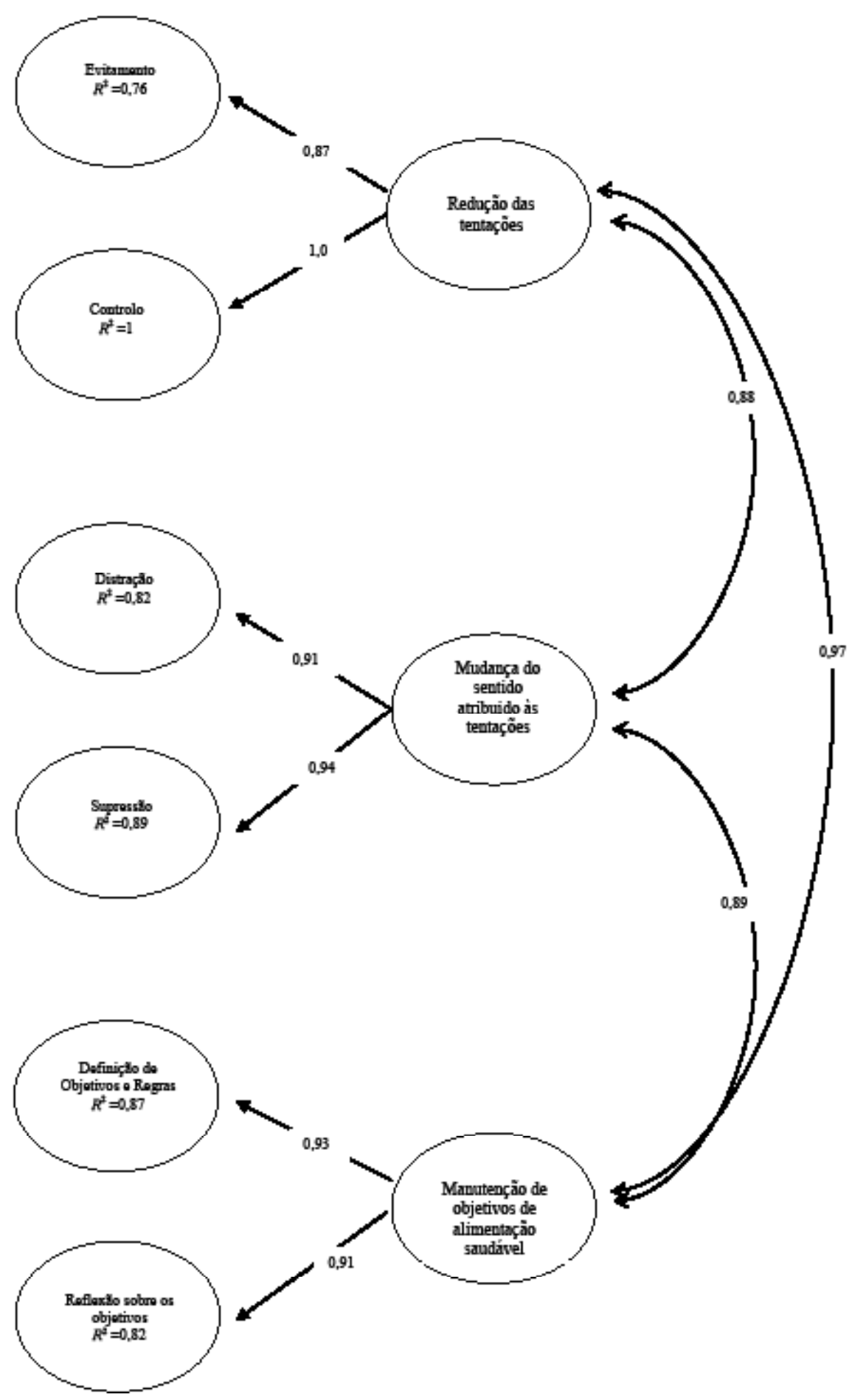

Figura 2. Análise fatorial confirmatória II. 
Gaspar, T., Tomé, G. Q., Simões, C. \& Matos, M. G. (2015). Estratégias de Auto-Regulação em Pré-Adolescentes e Adolescentes: Versão Portuguesa do TESQ-E.

Tabela 3

Variância Explicada $\left(R^{2}\right)$ e Erro Residual (Disturbance) dos Factores Dependentes - Análise Fatorial Confirmatória I e Análise Fatorial Confirmatória II

\begin{tabular}{ccc}
\hline & Análise Fatorial Confirmatória I & \\
\hline Factor & $R^{2}$ & Disturbance \\
\hline Evitamento & 0,65 & 0,59 \\
Controlo & 0,86 & 0,37 \\
Distração & 0,80 & 0,45 \\
Supressão & 0,86 & 0,38 \\
Definição de Objetivos e Regras & 0,85 & 0,39 \\
Reflexão sobre os Objetivos & 0,80 & 0,44 \\
& Análise Fatorial Confirmatória II & \\
Fator & $R^{2}$ & Disturbance \\
Evitamento & 0,76 & 0,49 \\
Controlo & 1,0 & 0,00 \\
Distração & 0,82 & 0,42 \\
Supressão & 0,89 & 0,33 \\
Definição de Objetivos e Regras & 0,87 & 0,35 \\
Reflexão sobre os Objetivos & 0,82 & 0,42 \\
\hline
\end{tabular}

Tabela 4

Correlações entre os Fatores Independentes

Reduzir Reduzir o Valor Apoiar o Objetivo da

Tentações Atribuido às Tentações Alimentação Saudável

Reduzir Tentações

Reduzir o Valor Atribuido às Tentações $\quad 0,88^{*}$

Apoiar o Objetivo da Alimentação Saudável $\quad 0,89^{*} \quad 0,97^{*}$

$* p \leq 0,05$.

\section{Correlações}

As correlações entre as três dimensões do TESQ-E variam entre 0,67 e 0,760 . As correlações entre os seis fatores do TESQ-E variam entre 0,47 (Evitamento e Reflexão sobre os objetivos) e 0,67 (entre Supressão e Distração).

Também é interessante a alta correlação entre os fatores de diferentes dimensões, tais como 0,62 (Reflexão sobre os objetivos e Distração) e 0,63 (Definição de objetivos e regras e Controlo) e de 0,63 (Definição de objetivos e regras e Distração) e 0,66 (Definição de objetivos e regras e Supressão).

\section{Diferenças entre Médias}

Foram analisadas as diferenças de Género e idade no questionário TESQ-E através da ANOVAs. Na Tabela 6 estão destacados em negrito o valor médio mais elevado (com significância estatística).

Foram encontradas diferenças de género significativas em quatro estratégias e duas dimensões Mudança do sentido atribuído às tentações e Manutenção de objetivos de alimentação saudável, e nas quatro estratégias correspondentes a estas dimensões. Em todos os casos, as meninas apresentaram médias mais elevadas de auto-regulação do que os rapazes.

Foram encontradas diferenças entre os grupos etários significativas em todas as 3 dimensões e em todas a estratégias correspondentes estas dimensões.

Em todos os casos, os pré-adolescentes apresentaram médias superiores de auto-regulação no comportamento alimentar do que os adolescentes. 
Psychology/Psicologia: Reflexão e Crítica, 28(4), 649-658.

Tabela 5

Correlação de Pearson entre as Dimensões e as Estratégias

\begin{tabular}{|c|c|c|c|c|c|c|c|c|}
\hline Dimensões & 1 & 2 & 3 & 4 & 5 & 6 & 7 & 8 \\
\hline Evitamento & -- & & & & & & & \\
\hline Controlo & $0,618^{*}$ & -- & & & & & & \\
\hline Distração & $0,485^{*}$ & $0,571 *$ & -- & & & & & \\
\hline Supressão & $0,549 *$ & $0,609 *$ & $0,668 *$ & -- & & & & \\
\hline Definição de objetivos e regras & $0,516^{*}$ & $0,635^{*}$ & $0,633^{*}$ & $0,663 *$ & -- & & & \\
\hline Reflexão sobre os objetivos & $0,470 *$ & $0,592 *$ & $0,624^{*}$ & $0,610^{*}$ & $0,654^{*}$ & -- & & \\
\hline Redução das tentações & $0,907 *$ & $0,904 *$ & $0,588^{*}$ & $0,644 *$ & $0,641 *$ & $0,592 *$ & -- & \\
\hline Mudança do sentido atribuido às tentações & $0,566^{*}$ & $0,645^{*}$ & $0,915^{*}$ & $0,912 *$ & $0,710^{*}$ & $0,675^{*}$ & $0,674 *$ & -- \\
\hline Manutenção de objetivos de alimentação saudável & $0,534 *$ & $0,669 *$ & $0,691 *$ & $0,697^{*}$ & $0,903 *$ & $0,917^{*}$ & $0,670^{*}$ & $0,760 *$ \\
\hline
\end{tabular}

Nota. *Correlação significativa 0,01 (2-tailed).

Tabela 6

Comparação de Género e Idade - Média, Desvio Padrão e ANOVAs - 6 Estratégias e 3 e Dimensões da TESQ-E

\begin{tabular}{lcccccccc}
\hline Estratégias/ Dimensões & \multicolumn{3}{c}{ Rapazes } & & \multicolumn{2}{c}{ Meninas } & \multicolumn{2}{c}{$F$} \\
\cline { 2 - 8 } & $N$ & $M$ & $D P$ & $N$ & $M$ & $D P$ & $0,55^{(n s)}$ \\
\hline Evitamento & 567 & 2,23 & 0,96 & 611 & 2,27 & 1,05 & $1,82^{(n s)}$ \\
Controlo & 564 & 2,44 & 0,97 & 608 & 2,52 & 1,03 & $4,01^{*}$ \\
Distração & 560 & 2,24 & 0,93 & 596 & $\mathbf{2 , 3 5}$ & 0,98 & \\
Supressão & 557 & 2,18 & 0,93 & 596 & $\mathbf{2 , 2 9}$ & 0,95 & $4,53^{*}$ \\
Definição de objectivos e regras & 561 & 2,22 & 1,01 & 598 & $\mathbf{2 , 3 7}$ & 1,03 & $6,29 *$ \\
Reflexão sobre os objectivos & 566 & 2,54 & 1,07 & 608 & $\mathbf{2 , 7 9}$ & 1,12 & $15,85^{* * *}$ \\
Redução das tentações & 567 & 2,33 & 0,87 & 611 & 2,38 & 1,00 & $0,97(n s)$ \\
Mudança do sentido atribuido às tentações & 560 & 2,20 & 0,84 & 597 & $\mathbf{2 , 3 2}$ & 0,89 & $5,26^{*}$ \\
Manutenção de objetivos de alimentação saudável & 569 & 2,38 & 0,97 & 610 & $\mathbf{2 , 5 8}$ & 0,97 & $12,12^{* *}$ \\
\hline
\end{tabular}

Estratégias/Dimensões

Pré-adolescentes (9-12 anos) Adolescentes (13-17 anos) $\quad F$

\begin{tabular}{|c|c|c|c|c|c|c|c|}
\hline & & & & & & & \\
\hline & $N$ & $M$ & $D P$ & $N$ & $M$ & $D P$ & \\
\hline Evitamento & 590 & 2,41 & 0,99 & 588 & 2,09 & 1,01 & $29,29 * * *$ \\
\hline Controlo & 588 & 2,61 & 1,02 & 584 & 2,35 & 0,96 & $20,23 * * *$ \\
\hline Distração & 576 & 2,43 & 0,99 & 580 & 2,17 & 0,91 & $21,70 * * *$ \\
\hline Supressão & 574 & 2,37 & 0,99 & 579 & 2,11 & 0,88 & $20,98 * * *$ \\
\hline Definição de objectivos e regras & 577 & 2,48 & 1,06 & 582 & 2,12 & 0,96 & $36,24 * * *$ \\
\hline Reflexão sobre os objectivos & 593 & 2,84 & 1,12 & 581 & 2,49 & 1,06 & $31,22 * * *$ \\
\hline Redução das tentações & 590 & 2,51 & 0,89 & 588 & 2,21 & 0,96 & $30,12 * * *$ \\
\hline Mudança do sentido atribuido às tentações & 577 & 2,39 & 0,91 & 580 & 2,14 & 0,81 & $25,76 * * *$ \\
\hline Manutenção de objetivos de alimentação saudável & 595 & 2,66 & 0,99 & 584 & 2,30 & 0,91 & $42,49 * * *$ \\
\hline
\end{tabular}

Nota. Em negrito os valores médios mais elevados (com significância estatística). *0,005-0,05;**0,001-0,005; ***0,000; (ns) = Não significativo $(>0,05)$. 
Gaspar, T., Tomé, G. Q., Simões, C. \& Matos, M. G. (2015). Estratégias de Auto-Regulação em Pré-Adolescentes e Adolescentes: Versão Portuguesa do TESQ-E.

\section{Discussão/Conclusão}

O objectivo principal do presente artigo foi estudar a validação da versão Portuguesa do TESQ-E. Este instrumento avalia as estratégias de auto-regulação do comportamento alimentar em pré-adolescentes e adolescentes. As estratégias de auto-regulação parecem envolver competências cognitivas, comportamentais e emocionais (De Ridder \& de Wit, 2006).

No presente estudo, pretendeu-se manter a estrutura fatorial inicial proposta pelos autores (De Vet et al., 2014; De Vet et al., 2013; Stok et al., 2013): 3 dimensões com 2 tipos de estratégias para cada dimensão, a escala TESQ-E mostrou uma boa consistência interna para todos os três fatores de ordem superior (Dimensões) sendo a dimensão mais referida pelos participantes a Manutenção de objectivos de alimentação saudável, e a menos referida a, Mudança do sentido atribuido às tentações e, considerando as estratégias, a estratégia mais referida foi a Reflexão sobre os objetivos e a menos referida a Supressão.

Testaram-se dois modelos de Análise fatorial confirmatória, um modelo com seis fatores correspondendo as seis estratégias e um modelo de segunda ordem onde, os seis fatores correspondendo a seis estratégias se agruparam em 3 dimensões, tal como no modelo original (De Vet et al., 2014; Stok et al., 2013). Ambos os modelos apresentaram bons níveis de adequação.

Foram encontradas diferenças de Género e idade no instrumento TESQ-E. Foram encontradas diferenças de género significativas em quatro estratégias e duas dimensões Mudança do sentido atribuído às tentações e Manutenção de objetivos de alimentação saudável, e nas quatro estratégias correspondentes a estas dimensões. Em todos os casos, as meninas apresentaram médias mais elevadas de auto-regulação do que os rapazes.

Foram encontradas diferenças entre os grupos etários significativas em todos as as 3 dimensões e em todas a estratégias correspondentes estas dimensões. Em todos os casos, os pre-adolescentes apresentaram médias superiores de auto-regulação no comportamento alimentar do que os adolescentes. Aauto-regulação é uma competência que se desenvolve gradualmente durante a infância e a adolescência (Sokol et al., 2013).

Os resultados encontrados na versão Portuguesa vão no mesmo sentido do que os encontrados na análise da versão original (De Vet et al., 2014; De Vet et al., 2013; Stok et al., 2013). A versão Portuguesa do instrumento TESQ-E, mantendo a estrutura da versão original, apresenta boas propriedades métricas e é sensivel às diferenças de género e idade.

O TESQ-E é pois um instrumento válido na avaliação da auto-regulação do comportamento alimentar em pré-adolescentes e adolescentes, e um importante contributo para a intervenção e investigação no âmbito da promoção de saúde e prevenção da doença em diversas áreas disciplinares, da Educação à Saúde.

\section{Referências}

Abraham, C., Norman, P., \& Conner, M. (2000). Towards a psychology of health-related behaviour change. In P. Norman, C. Abraham, \& M. Conner (Eds.), Understanding and changing health behaviour: From health beliefs to self-regulation (pp. 343-369). Amsterdam, Holland: Hardwood.

Abraham, C., \& Sheeran, P. (2000). Understanding and changing health behaviour: From health beliefs to self-regulation. In P. Norman, C. Abraham, \& M. Conner (Eds.), Understanding and changing health behaviour: From health beliefs to selfregulation (pp. 3-24). Amsterdam, Holland: Hardwood.

Baumeister, R. F. (1999). The nature and structure of the self: An overview. In R. F. Baumesteir (Ed.), The self in social psichology (pp. 1-24). Philadelphia, PA: Psychology Press.

Baumeister, R. F. (2005). The cultural animal: Human nature, meaning and social life. New York: Oxford University Press.

Baumeister, R. F., \& Vohs, K. D. (2004). Self-regulation. In C. Perterson \& M. E. P. Seligman (Eds.), Character strengths and virtues: A handbook and classification (pp. 499-516). New York: Oxford Press.

Bentler, P. M. (1995). EQS structural equations program manual. Encino, CA. Multivariate Software.

Cameron, L. D., \& Leventhal, H. (2003). Self-regulation, health and illness: An overview. In L. D. Cameron \& H. Leventhal (Eds.), The self-regulation of health and illness behaviour (pp. 1-13). London: Routloedge.

De Ridder, D., \& de Wit, J. (2006). Self-regulation in health behaviour: Concepts, theories and central issues. In D. De Ridder \& J. de Wit (Eds.), Self-regulation in health behaviour (pp. 3-23). London: John Wiley \& Sons.

De Vet, E., De Ridder, D., Stok, M., Brunso, K., Baban, A., \& Gaspar, T. (2014). Assessing self-regulation strategies: Development and validation of the TEMPEST Self-Regulation Questionnaire for Eating (TESQ_E) in Adolescents. International Journal of Behavioral Nutrition and Physical Activity, 11, 106. doi:10.1186/s12966-014-0106-Z

De Vet, E., de Wit, J., Luszczynska, A., Stok, F. M., Gaspar T., Pratt, M., ...de Ridder, D. (2013). Access to excess: How do adolescents deal with unhealthy foods in their environment? European Journal of Public Health, 23(5), 752-756. doi:10.1093/eurpub/cks185

Duckworth, A. L., \& Seligman, M. E. (2006). Self-discipline outdoes IQ in predicting academic performance of adolescents. Psychological Science, 16, 939-944.

Gaspar, T., Matos, M. G., Luszczynska, A., Baban, A., \& de Wit, J. (2014). The impacto of a rural and urban context in eating awareness and self-regulation strategies in children and adolescents from eight European countries. International Journal of Psychology, 49(3), 158-166. doi:10.1002/ijop.12046

Gebhardt, W. A. (2006). Contextualizing health behaviors: The role of personal goals. In D. De Ridder \& J. de Wit (Eds.), Self-regulation in health behaviour (pp. 27-43). London: John Wiley \& Sons.

Gestsdottir, S., \& Lerner, R. M. (2008). Positive development in adolescence: The development and role of intentional selfregulation. Human Development, 51, 202-224.

Luszcznska, A., de Wit, J. B., de Vet, E., Januszewicz, A., Liszewska, N., Johnson, F., ...Stok, M. (2013). At-home environment, out-of-home environment, snacks and sweetened beverages intake in preadolescence, early and mid-adolescence: The interplay between environment and self-regulation Journal of Youth and Adolescence, 42(12), 1873-1883. doi:10.1007/s10964-013-9908-6 
Sokol, B., Grouzet, F., \& Muller, U. (2013). Self-regulation and autonomy: Social developmental dimensions of human conduct. New York: Cambridge University Press.

Stok, M., De Ridder, D., de Vet, E., de Wit, J., \& the Tempest Consortium. (2013). The TEMPEST Handbook: Strategies to support healthy eating in adolescents. The Hague, Netherlands: Dutch Nutrition Centre.

Vohs, K. D., \& Baumeister, R. F. (2004). Understanding selfregulation: An introduction. In R. F. Baumeister \& K. D. Vohs (Eds.), Handbook of self regulation: Research, theory, and applications (pp. 1-9). New York: Guilford Press.

Zimmerman, J. (2002). Achieving academic excellence: A self-regulatory perspective. In M. Ferrari (Ed.), The pursuit of excellence through education (pp. 85-110). Mahwah, NJ: Erlbaum.

Zeidner, M., Boekaerts, M., \& Pintrich, P. R. (2000). Self-regulation: Directions and challenges for future research. In M. Boekaerts, P. R. Pintrich, \& M. Zeidner (Eds.), Handbook of self-regulation (pp. 750-768). San Diego, CA: Academic Press. 